\title{
Search Strategy for Sleptons and Dark Matter Using the LHC as a Photon Collider
}

\author{
Lydia Beresford" and Jesse Liu $\oplus^{\dagger}$ \\ Department of Physics, University of Oxford, Oxford OX1 3RH, United Kingdom
}

(Received 3 December 2018; revised manuscript received 22 July 2019; published 3 October 2019)

\begin{abstract}
We propose a search strategy using the LHC as a photon collider to open sensitivity to scalar lepton (slepton $\tilde{\ell}$ ) production with masses around 15 to $60 \mathrm{GeV}$ above that of neutralino dark matter $\tilde{\chi}_{1}^{0}$. This region is favored by relic abundance and muon $(g-2)_{\mu}$ arguments. However, conventional searches are hindered by the irreducible diboson background. We overcome this obstruction by measuring initial state kinematics and the missing momentum four-vector in proton-tagged ultraperipheral collisions using forward detectors. We demonstrate sensitivity beyond LEP for slepton masses of up to $200 \mathrm{GeV}$ for $15 \lesssim \Delta m\left(\tilde{\ell}, \tilde{\chi}_{1}^{0}\right) \lesssim 60 \mathrm{GeV}$ with $100 \mathrm{fb}^{-1}$ of $13 \mathrm{TeV}$ proton collisions. We encourage the LHC collaborations to open this forward frontier for discovering new physics.
\end{abstract}

DOI: 10.1103/PhysRevLett.123.141801

Introduction.-Elucidating the elementary properties of dark matter $(\mathrm{DM})$ is among the most urgent problems in fundamental physics. The lightest neutralino $\tilde{\chi}_{1}^{0}$ in supersymmetric (SUSY) extensions of the standard model (SM) is one of the most motivating DM candidates [1-3]. A favored scenario involves scalar partners of the charged leptons (sleptons $\tilde{\ell}$ ) being 1 to tens of $\mathrm{GeV}$ above the $\tilde{\chi}_{1}^{0}$ mass. This enables interactions that reduce the $\tilde{\chi}_{1}^{0}$ cosmological relic abundance to match the observed value [4] via a mechanism called slepton coannihilation $[5,6]$. Furthermore, partners of the muon (smuon $\tilde{\mu}$ ) and neutralinos with masses near the weak scale are a leading explanation for $3 \sigma$ to $4 \sigma$ deviations between measurements of the muon magnetic moment and SM prediction [7-10].

Remarkably, Large Hadron Collider (LHC) searches for these key targets have no sensitivity when mass differences are $15 \lesssim \Delta m\left(\tilde{\ell}, \tilde{\chi}_{1}^{0}\right) \lesssim 60 \mathrm{GeV}$ [11-14]. Here, Large Electron Positron (LEP) collider limits remain the most stringent, excluding $m(\tilde{\ell}) \lesssim 97 \mathrm{GeV}$ [15-17]. Sensitivity is hindered by an obstruction generic to all LHC search strategies for invisible DM states and their mediators [18-32]: the kinematics of colliding quarks and gluons are immeasurable. Without this initial state information, the missing momentum four-vector $p_{\text {miss }}$ left by DM can be determined only in the plane transverse to the beam $\left(\mathbf{p}_{T}^{\text {miss }}\right)$. This precludes direct DM mass reconstruction that would otherwise provide effective discrimination against neutrino $\nu$ backgrounds.

Published by the American Physical Society under the terms of the Creative Commons Attribution 4.0 International license. Further distribution of this work must maintain attribution to the author(s) and the published article's title, journal citation, and DOI. Funded by SCOAP ${ }^{3}$.
This Letter proposes a search strategy to resolve these long-standing problems by using the LHC as a photon collider [33]. In a beam crossing, protons can undergo an ultraperipheral collision (UPC), where photons from the electromagnetic fields interact to produce sleptons exclusively, $p p \rightarrow p(\gamma \gamma \rightarrow \tilde{\ell} \tilde{\ell}) p$. The sleptons decay as $\tilde{\ell} \rightarrow \ell \tilde{\chi}_{1}^{0}$, resulting in the very clean final state $p(2 \ell+$ $\left.p_{\text {miss }}\right) p$ of our search: two intact protons, two leptons $\ell$, and missing momentum (Fig. 1). As the beam energy is known, measuring the outgoing proton kinematics determines the colliding photon momenta and thus $p_{\text {miss }}$. This experimental possibility is opened by the ATLAS Forward Proton (AFP) [34] and CMS-TOTEM Precision Proton Spectrometer (CT-PPS) [35,36] forward detectors, which recorded their first data in 2017 and 2016, respectively. CMS-TOTEM moreover observed double lepton production in high-luminosity proton-tagged events [37], demonstrating that initial state reconstruction is feasible.

Photon collisions at the LHC reach sufficient rates to probe rare processes such as SM light-by-light scattering [38,39], anomalous gauge couplings [40,41], axionlike particles $[42,43]$, and dark sectors $[44,45]$. Nonetheless,

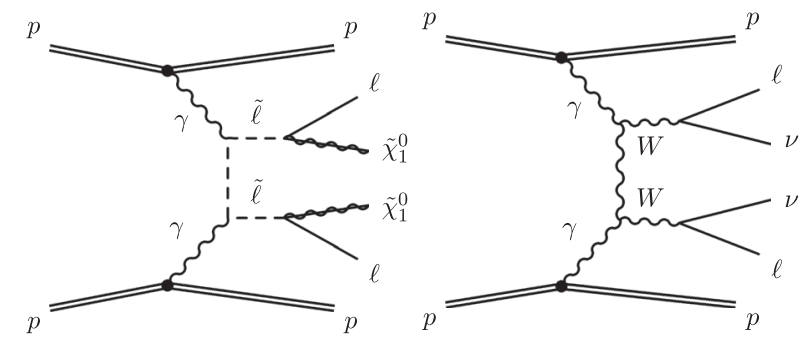

FIG. 1. Exclusive pair production of (left panel) scalar leptons ("sleptons") $\tilde{\ell}$ decaying to dark matter $\tilde{\chi}_{1}^{0}$ and (right panel) SM diboson $W W$ background using the LHC as a photon collider. 
it is widely considered that photon fusion production of sleptons is not competitive as a discovery window compared to electroweak production [11-14]; existing photon collider studies therefore focus on slepton mass measurement for specific benchmark points [46-50]. Our proposal argues to the contrary that photon collisions play an essential role in SUSY and DM searches. We emulate AFP and CT-PPS proton tagging, which enables powerful background suppression. We demonstrate a strategy that surpasses LEP sensitivity in the favored $15 \lesssim \Delta m\left(\tilde{\ell}, \tilde{\chi}_{1}^{0}\right) \lesssim$ $60 \mathrm{GeV}$ corridor, underscoring the importance of initial state kinematics and $p_{\text {miss }}$ for the LHC discovery program.

Photon collider simulation.-Electromagnetic fields surrounding ultrarelativistic protons can be modeled as a beam of nearly on-shell photons, which is known as the equivalent photon approximation [51]. We consider pair production of electrically charged particles $X$ via photon fusion $\gamma \gamma \rightarrow X X$. Analytic expressions of their QED cross sections $\sigma_{\gamma \gamma \rightarrow X X}$ may be found in Refs. [46,50,52,53]. The LHC cross section $\sigma_{\gamma \gamma \rightarrow X X}^{(p p)}$ is then the convolution of $\sigma_{\gamma \gamma \rightarrow X X}$ with the effective photon luminosity $L_{\gamma \gamma}^{(p p)}$ from the protons

$$
\sigma_{\gamma \gamma \rightarrow X X}^{(p p)}=\int \sigma_{\gamma \gamma \rightarrow X X}\left(m_{\gamma \gamma}\right) \frac{d L_{\gamma \gamma}^{(p p)}}{d m_{\gamma \gamma}} d m_{\gamma \gamma},
$$

where $m_{\gamma \gamma}$ is the invariant mass of the two-photon system. We use MadGraph v2.6.1 [54,55] to numerically evaluate Eq. (1) and perform Monte Carlo simulations for signal and background processes. Throughout, cross sections $\sigma_{\gamma \gamma \rightarrow X X}^{(p p)}$ refer to $p p \rightarrow p(\gamma \gamma \rightarrow X X) p$ processes with default generator preselections applied, except that the lepton $p_{T}$ requirement is removed. We study the resulting events using the PYLHE package [56] and parametrize the detector effects as follows.

The forward detectors identify both the intact outgoing protons at $z \simeq \pm 220 \mathrm{~m}$ downstream from the collision point and measure their energies $E_{\text {forward }}$. Protons are steered outside the beam profile by the LHC dipole magnets due to the fractional energy loss $\xi=1-$ $E_{\text {forward }} / E_{\text {beam }}$ relative to the beam energy $E_{\text {beam }}=$ 6.5 TeV. The AFP and CT-PPS proton acceptance is close to $100 \%$ for $0.02<\xi<0.12$ [34-36], which we emulate by requiring emitted photon energies $130<E_{\gamma}<780 \mathrm{GeV}$. The Supplemental Material [57] validates the finding that the proton $p_{T}$ is very small, which is neglected in MadGraph, and also shows the impact of raising the minimum $\xi$ to $0.025\left(E_{\gamma}>162.5 \mathrm{GeV}\right)$. Existing studies typically assume $100 \%$ survival probability $P_{\text {survival }}^{(p p)}$ of a proton remaining intact following photon emission [46-50], but phenomenological studies suggest lower values for the range of $E_{\gamma}$ considered [58,59]. We estimate $P_{\text {survival }}^{(p p)}$ using SuperChic 3.02 [60], which we treat as an efficiency parametrized by $P_{\text {survival }}^{(p p)}=a \exp \left(-b m_{\gamma \gamma}\right)$, where $a=0.988$, $b=4.67 \times 10^{-4}$; see the Supplemental Material [57] for the origin of this parametrization. This gives $P_{\text {survival }}^{(p p)}=$ $94 \%$ for $m_{\gamma \gamma}=100 \mathrm{GeV}$ and falls to $62 \%$ for $m_{\gamma \gamma}=1000 \mathrm{GeV}$. We conservatively smear the photon four-vector $p_{\gamma}^{\text {smeared }}=p_{\gamma}^{\text {generated }} G_{\gamma}\left(1, \sigma_{\gamma}\right)$ using a Gaussian $G_{\gamma}$ with width $\sigma_{\gamma}=5 \%$, based on the AFP resolution of $5 \mathrm{GeV}$ at $\xi \simeq 0.02$ [34].

The central detectors reconstruct isolated leptons (electrons $e$ and muons $\mu$ throughout). To emulate detector resolution, we smear the lepton momenta $p_{\ell}$ using a Gaussian $G_{\ell}$ with $p_{T}$-dependent width $\sigma_{\ell}\left(p_{T}\right)$. We extract $\sigma_{\ell}$ from Refs. [61,62], which are predominantly below 5\% for the relevant range of $p_{T}$ and have minimal impact on the results. We parametrize $p_{T}$-dependent reconstruction efficiencies in accord with ATLAS [14], which accounts for all lepton quality conditions. This requires that leptons satisfy transverse momentum $p_{T}^{e(\mu)}>4.5(4) \mathrm{GeV}$ and pseudorapidity $\left|\eta_{\ell}\right|<2.5$.

To simulate the simplified model signal $\gamma \gamma \rightarrow \tilde{\ell} \tilde{\ell}$, we employ the model specified by the SUSY Les Houches Accord parameter file from the auxiliary material of Ref. [14]. This allows comparisons with existing LHC constraints. Only sleptons $\tilde{\ell}$ and the stable neutralino $\tilde{\chi}_{1}^{0}$, whose masses are free parameters, are kinematically accessible. A fourfold mass degeneracy is assumed such that scalar partners of the left-handed and right-handed electrons and muons (selectrons $\tilde{e}$ and smuons $\tilde{\mu}$ ) satisfy $m\left(\tilde{\ell}_{L, R}\right)=m\left(\tilde{e}_{L}\right)=m\left(\tilde{e}_{R}\right)=m\left(\tilde{\mu}_{L}\right)=m\left(\tilde{\mu}_{R}\right)$. The sleptons decay $\tilde{\ell} \rightarrow \ell \tilde{\chi}_{1}^{0}$ with $100 \%$ branching ratio and are handled by MadGraph. All other SUSY states are kinematically inaccessible with masses well above $10 \mathrm{TeV}$. We sample $m(\tilde{\ell})$ in $25 \mathrm{GeV}$ steps, and $\Delta m\left(\tilde{\ell}, \tilde{\chi}_{1}^{0}\right)$ in steps of no more than $20 \mathrm{GeV}$. We simulate $50 \times 10^{3}$ events per mass point and normalize to cross sections calculated in MadGraph, which are consistent with those obtained in Refs. [49,50]. For $m(\tilde{\ell})=100 \mathrm{GeV}$, the cross section $\sigma_{\gamma \gamma \rightarrow \tilde{\ell} \tilde{\ell}}^{(p p)}$ is $2.5 \mathrm{fb}$ and falls to $0.25 \mathrm{fb}$ for $m(\tilde{\ell})=200 \mathrm{GeV}$. Only the first two generations $\tilde{\ell} \in[\tilde{e}, \tilde{\mu}]$ are considered; study of scalar partners of $\tau$ leptons (staus $\tilde{\tau}$ ) are deferred to future work.

Search strategy.-Our search strategy focuses on extracting the signal from the dominant irreducible $\gamma \gamma \rightarrow$ $W W \rightarrow \ell \nu \ell \nu$ background. The $W W$ cross section times the dileptonic branching fraction $\mathcal{B}$ is $\sigma_{\gamma \gamma \rightarrow W W}^{(p p)} \times \mathcal{B} \simeq 5 \mathrm{fb}$, which is comparable in size to the slepton signals. We generate $50 \times 10^{3}$ events of this process using MadGraph, which also handles the decays to preserve spin correlations of the leptons. We use dilepton triggers for event selection, which we emulate using a $p_{T}^{\ell}>15 \mathrm{GeV}$ condition. Requiring same flavor leptons (ee or $\mu \mu)$ halves the $W W$ background while preserving signal. We then reconstruct three defining features that characterize the signals and background to optimize search sensitivity: mediator mass $(W$ or $\tilde{\ell})$, invisible mass $\left(\nu\right.$ or $\left.\tilde{\chi}_{1}^{0}\right)$, and mediator spin. 

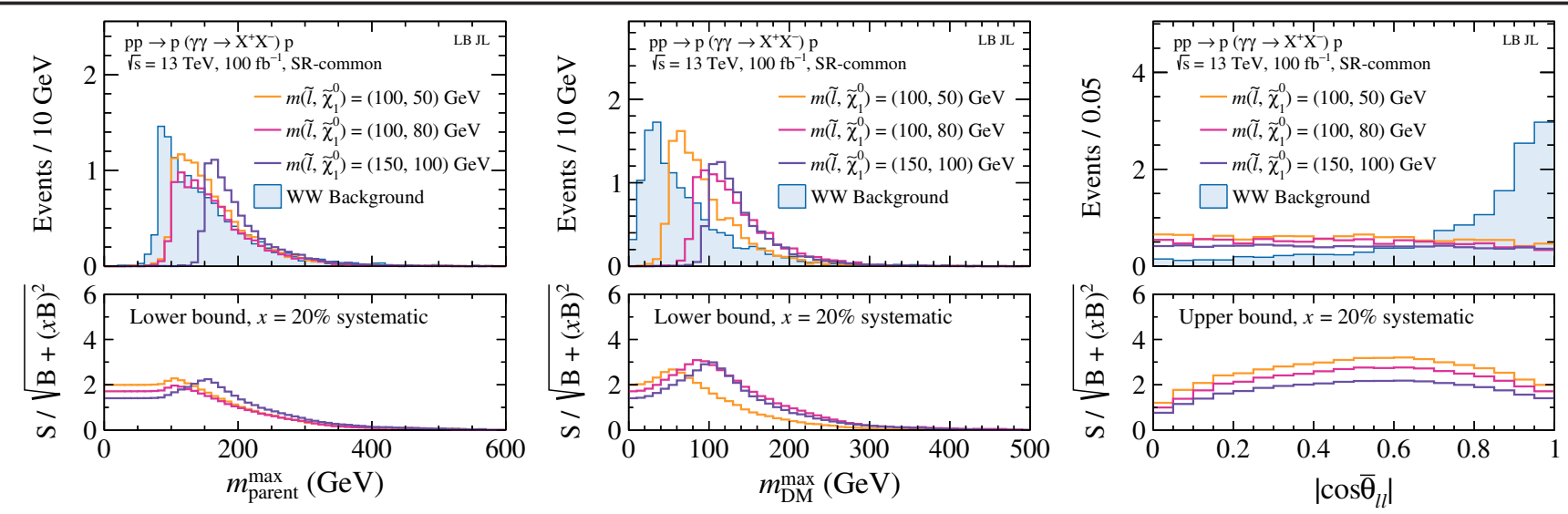

FIG. 2. Kinematic distributions of discriminants reconstructing the mass and spin for benchmark slepton signals (lines) and $W W$ background (filled areas), normalized to $100 \mathrm{fb}^{-1}$. Proton survival, $\xi$ acceptance, lepton efficiencies, and detector smearing are applied, but no lepton trigger emulation is imposed. The event selection applied, denoted SR-common, requires $m_{\mathrm{DM}}^{\max }>0 \mathrm{GeV}$, $\left|\eta_{\ell}\right|<2.5$, same flavor leptons, and $m_{T 2}^{0}>2 \mathrm{GeV}$. The legend displays signal masses. The lower panels estimate the statistical significance after integrating the signal $S$ and background $B$ counts with the indicated bound on the variable.

At the LHC, proton tagging enables unambiguous bounds on both the parent mediator and DM masses. The mass of the $\tilde{\ell}$ mediators is bound by the invariant mass of the initial state two-photon system $m_{\gamma \gamma}^{2}=$ $\left(p_{\gamma_{1}}+p_{\gamma_{2}}\right)^{2} \geq\left(2 m_{\tilde{\ell}}\right)^{2}$. The Supplemental Material [57] shows that signals have broad tails in $m_{\gamma \gamma}$, allowing AFP and CT-PPS acceptance even for low masses $m(\tilde{\ell}) \lesssim$ $150 \mathrm{GeV}$. Meanwhile, the invariant mass of the invisible system $W_{\text {miss }}$ bounds the DM masses $W_{\text {miss }}^{2}=p_{\text {miss }}^{2} \geq$ $\left(2 m_{\tilde{\chi}_{1}^{0}}\right)^{2}$. Here, $p_{\text {miss }}=\sum_{i} p_{i}-\sum_{f} p_{f}$ is the vectorial sum of the momenta of the visible final states $p_{f}$ subtracted from the initial states $p_{i}$. In this search, we have $\sum_{i} p_{i}=$ $p_{\gamma_{1}}+p_{\gamma_{2}}$ and $\sum_{f} p_{f}=p_{\ell_{1}}+p_{\ell_{2}}$. We find the ratio $m_{\gamma \gamma} / W_{\text {miss }}$ to be useful for $\Delta m\left(\tilde{\ell}, \tilde{\chi}_{1}^{0}\right) \lesssim 30 \mathrm{GeV}$ signals.

To improve mass reconstruction of the parent mediator and DM states, one can impose hypotheses about the decay topology. Assuming the symmetric pair of semi-invisible decays $\tilde{\ell} \tilde{\ell} \rightarrow \ell \tilde{\chi}_{1}^{0} \ell \tilde{\chi}_{1}^{0}$, with photon and lepton momenta measured, results in the Harland-Lang-Kom-SakuraiStirling variables defined in Ref. [50]. These also provide mass bounds on the parent mediator $m_{\text {parent }}^{\max } \geq m(\tilde{e})$ and invisible system $m_{\mathrm{DM}}^{\max } \geq m\left(\tilde{\chi}_{1}^{0}\right)$. Importantly, these variables have more steeply falling tails than $m_{\gamma \gamma}$ and $W_{\text {miss }}$, respectively, and therefore provide better signal separation from the $W W$ background.

To exploit the mediator spin, we use the Barr-Melia variable $[63,64]$ defined by $\cos \bar{\theta}_{\ell \ell}=\tanh \left[\frac{1}{2}\left(\bar{\eta}_{\ell_{1}}-\bar{\eta}_{\ell_{2}}\right)\right]$, where the pseudorapidities $\bar{\eta}$ are evaluated in the dilepton center-of-mass frame (denoted by overlines). Leptons from spin $0 \tilde{\ell}$ mediators decay more centrally than those from spin $1 \mathrm{~W}$ bosons, offering discrimination power.

Figure 2 displays distributions of benchmark signals and the $W W$ background for these mass and spin sensitive variables, normalized to $100 \mathrm{fb}^{-1}$. From this, we impose $\left|\cos \bar{\theta}_{\ell \ell}\right|<0.65$ and construct three signal region (SR) categories targeting small "compressed," medium "corridor," and large mass differences $\Delta m\left(\tilde{\ell}, \tilde{\chi}_{1}^{0}\right)$ : (1) SR-compressed- $m_{\text {parent }}^{\max }>80 \mathrm{GeV}, \quad m_{\mathrm{DM}}^{\max }>0 \mathrm{GeV}$, $m_{\gamma \gamma} / W_{\text {miss }}<1.5$; (2) $\quad$ SR-corridor $-m_{\text {parent }}^{\max }>120 \mathrm{GeV}$, $m_{\mathrm{DM}}^{\max }>80 \mathrm{GeV}$; and (3) SR-large $-m_{\mathrm{parent}}^{\max }>130 \mathrm{GeV}$, $m_{\mathrm{DM}}^{\max }>20 \mathrm{GeV}$. An improved strategy would involve a shape analysis of $\left(m_{\mathrm{parent}}^{\max }, m_{\mathrm{DM}}^{\max }\right)$ akin to a bump hunt [29] in two dimensions, but it is deferred to future work.

Other potential irreducible processes include $\tau \tau \rightarrow$ $\ell \nu \nu \ell \nu \nu$, which has a large rate $\sigma_{\gamma \gamma \rightarrow \tau \tau}^{(p p)} \times \mathcal{B} \simeq 74 \times 0.35^{2} \simeq$ $9.1 \mathrm{pb}$. We reject this process by reconstructing the $\tau$ mass end point using the stransverse mass $m_{T 2}^{\chi=0}>2 \mathrm{GeV}$ (see Refs. [65-67] for a definition). This variable uses the lepton momenta and missing transverse momentum defined by $\mathbf{p}_{T}^{\text {miss }} \equiv-\mathbf{p}_{T}^{\ell_{1}}-\mathbf{p}_{T}^{\ell_{2}}$. As we use this variable to reject $\tau$ 's decaying to massless neutrinos, we set the hypothesized mass of the invisible state $\chi=0 \mathrm{GeV}$ in $m_{T 2}^{\chi=0}$ throughout. We validate mitigation of this background by generating an event sample in MadGraph using the sm-lepton masses model to decay the $\tau$ 's. The Supplemental Material [57] shows that this requirement has a signal efficiency above $95 \%$ for the target mass points $\Delta m\left(\tilde{\ell}, \tilde{\chi}_{1}^{0}\right) \gtrsim 20 \mathrm{GeV}$. Top quark pairs $\gamma \gamma \rightarrow t \bar{t} \rightarrow b \ell \nu b \ell \nu$ contribute a small rate $\sigma_{\gamma \gamma \rightarrow t \bar{t}}^{(p p)} \times \mathcal{B} \simeq 0.33 \times 0.21^{2} \simeq 0.015 \mathrm{fb}$, and we assume that a jet veto renders this background negligible.

Turning to reducible backgrounds induced by detector misreconstruction, these typically require data driven techniques to estimate reliably. We briefly discuss possible mitigation strategies. First, nonresonant lepton pairs $\gamma \gamma \rightarrow \ell \ell$, where $\ell \in[e, \mu]$, have a large cross section $\sigma_{\gamma \gamma \rightarrow e e}^{(p p)}=\sigma_{\gamma \gamma \rightarrow \mu \mu}^{(p p)}=60 \mathrm{pb}$. Missing momentum results solely from detector resolution, and this background is 
also rendered negligible by the $m_{T 2}$ requirement. This also suppresses resonant dilepton decays of quarkonia such as $J / \psi$ and $\Upsilon$ states. Next, fake and nonprompt leptons, such as semileptonic decays of $B$ hadrons, typically become significant at low lepton $p_{T}$ [14]. We expect these to be well controlled by standard lepton quality requirements. Indeed fake leptons are negligible in current slepton searches using lepton triggers [11-13].

Finally, pileup collisions can fake intact UPC protons when occurring in the same event as an exclusive or nonexclusive process with two leptons and $p_{\text {miss }}$. Robustly estimating these pileup backgrounds requires data driven methods. We suggest mitigation strategies for dedicated study in the experimental collaborations, with further discussion given in the Supplemental Material [57]. To suppress nonexclusive processes, recent analyses veto tracks within $1 \mathrm{~mm}$ of the dilepton vertex [68-70]. This can be optimized further, such as by lowering track $p_{T}$ thresholds down to $100 \mathrm{MeV}$ [39]. Requiring low activity in the zero degree calorimeters [71] could also suppress nonexclusive processes, with ongoing developments of radiation hard technologies for high-luminosity runs [72]. Measuring arrival time differences of forward protons with target resolutions of $10 \mathrm{ps}$ [73-76] allows us to match with lepton vertices. Assuming a conservative 30 ps resolution, Ref. [76] finds a 1 order of magnitude background rejection for $90 \%$ signal efficiency, while early measurements using LHC run 2 data already reach 20 ps [77]. Further requirements could enhance signal discrimination, such as imposing low $p_{T}$ forward protons, and correlating lepton and proton kinematics using multivariate techniques.

Sensitivity and discussion.-We now evaluate the sensitivity of our search strategy for the slepton-DM simplified model. We assume two benchmark luminosities $\mathcal{L}=100(300) \mathrm{fb}^{-1}$, which correspond to the cumulative dataset for LHC run 2 (3). We use the asymptotic Poisson significance with uncertain background $Z_{A}\left(S, B, \sigma_{B}\right)$ $[78,79]$. This takes as input the signal $S$, background $B$ counts, and we ascribe a background systematic uncertainty of $\sigma_{B}=0.2 B$. For $100 \mathrm{fb}^{-1}$, SR compressed has $B=0.47$, and the highest $S=5$ is for the $m\left(\tilde{\ell}, \tilde{\chi}_{1}^{0}\right)=(100,80) \mathrm{GeV}$ signal. This corresponds to a signal efficiency of $2 \%$ with respect to the generated cross section, and a significance of $3.3 \sigma$, rising to $5.7 \sigma$ for $300 \mathrm{fb}^{-1}$. Meanwhile, SR corridor targets slightly larger $\Delta m\left(\tilde{\ell}, \tilde{\chi}_{1}^{0}\right)$, where $B=0.74$ and the highest $S=5.5$ corresponds to the $m\left(\tilde{\ell}, \tilde{\chi}_{1}^{0}\right)=$ $(125,80) \mathrm{GeV}$ signal, translating to $3.3 \sigma$ significance, rising to $5.7 \sigma$ for $300 \mathrm{fb}^{-1}$. SR large probes larger $\Delta m\left(\tilde{\ell}, \tilde{\chi}_{1}^{0}\right)$, with $B=1.2$ at $100 \mathrm{fb}^{-1}$ and the highest $S=$ 5.9 is for the $m\left(\tilde{\ell}, \tilde{\chi}_{1}^{0}\right)=(125,40) \mathrm{GeV}$ signal, corresponding to a significance of $3.1 \sigma$, rising to $5.4 \sigma$ for $300 \mathrm{fb}^{-1}$. The Supplemental Material [57] presents cutflows showing the yields for benchmark signals and the $W W$ background sequentially after each requirement.

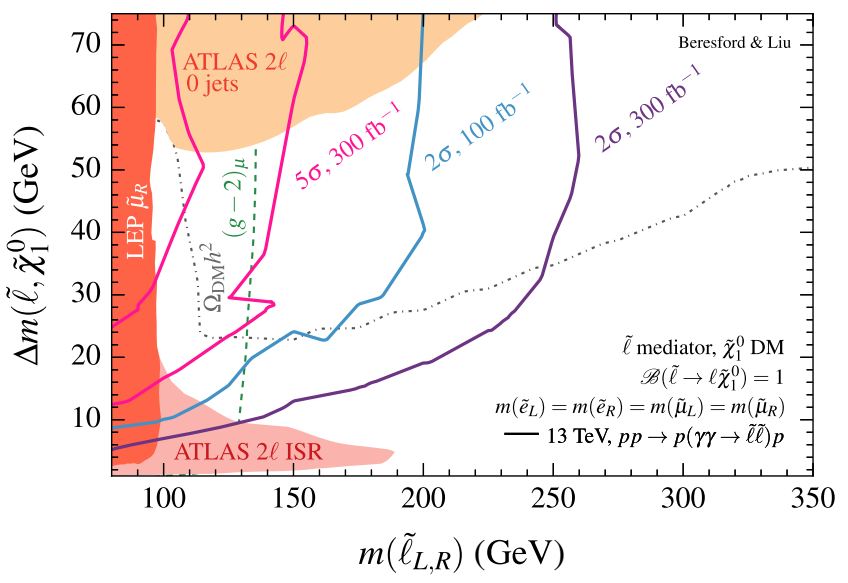

FIG. 3. Projected photon collider sensitivity of $\gamma \gamma \rightarrow \tilde{\ell} \tilde{\ell}$ using $13 \mathrm{TeV}$ proton-tagged LHC collisions. Solid lines (this Letter) show the $2 \sigma$ sensitivity contours for integrated luminosities of $100 \mathrm{fb}^{-1}$ (blue) and $300 \mathrm{fb}^{-1}$ (purple), along with $5 \sigma$ at $300 \mathrm{fb}^{-1}$ (pink). A simplified model of slepton mediators $\tilde{\ell}$ with a fourfold mass degeneracy decaying to neutralino $\mathrm{DM} \tilde{\chi}_{1}^{0}$ is considered. Filled regions denote constraints from ATLAS $2 \ell 0$ jets $[11,12]$ (yellow), $2 \ell$ ISR searches [14] (pink), and LEP for partners of the right-handed muons $\tilde{\mu}_{R}$ [15-17] (orange). Dashed lines indicate parameter space favored by relic abundance $\Omega_{\mathrm{DM}} h^{2}$ [4] (gray) and muon $(g-2)_{\mu}$ [8] (green) measurements, computed using MICROMEGAs [80].

Figure 3 shows the $2 \sigma$ "sensitivity" contours of our search strategy (the solid lines) in the $\Delta m\left(\tilde{\ell}, \tilde{\chi}_{1}^{0}\right)$ vs $m(\tilde{\ell})$ plane, with $5 \sigma$ "discovery" contours displayed for $300 \mathrm{fb}^{-1}$. For each signal point, we use the highest significance out of the three SRs. Our strategy unambiguously surpasses the existing collider sensitivity (the filled regions) in the $15 \lesssim \Delta m\left(\tilde{\ell}, \tilde{\chi}_{1}^{0}\right) \lesssim 60 \mathrm{GeV}$ corridor. For $\Delta m\left(\tilde{\ell}, \tilde{\chi}_{1}^{0}\right) \sim 40 \mathrm{GeV}, 2 \sigma$ sensitivity reaches $m(\tilde{e}) \sim$ $200(250) \mathrm{GeV}$ for $100(300) \mathrm{fb}^{-1}$, while $5 \sigma$ sensitivity extends up to $m(\tilde{\ell}) \sim 140 \mathrm{GeV}$ using $300 \mathrm{fb}^{-1}$.

The mass reach depends on several factors. As $m(\tilde{\ell})$ increases, the $\gamma \gamma \rightarrow \tilde{\ell} \tilde{\ell}$ cross section decreases and the search becomes statistically limited. However, signals with larger $m(\tilde{\ell})$ are easier to distinguish from the $W W$ background as the signal becomes better separated from the $W$ boson mass; higher DM masses are similarly easier to separate. For $m(\tilde{\ell}) \lesssim 120 \mathrm{GeV}$, sensitivity is limited by the forward detector acceptance.

The canonical LHC search for sleptons employs the " $2 \ell 0$ jets" signature, where the ATLAS $8 \mathrm{TeV}, 20.3 \mathrm{fb}^{-1}$ analysis gives the most stringent limit for $m(\tilde{\ell}) \lesssim 250 \mathrm{GeV}$ [11]. Notably, the $13 \mathrm{TeV}, 36.1 \mathrm{fb}^{-1}$ counterpart [12] did not surpass the $8 \mathrm{TeV}$ analysis sensitivity for $\Delta m\left(\tilde{\ell}, \tilde{\chi}_{1}^{0}\right) \lesssim 60 \mathrm{GeV}$, despite higher center-of-mass energy and luminosity, with similar results from CMS [13].

Our strategy has limited sensitivity to the compressed region $\Delta m\left(\tilde{\ell}, \tilde{\chi}_{1}^{0}\right) \lesssim 10 \mathrm{GeV}$ due to the trigger emulation 
$p_{T}^{\ell}>15 \mathrm{GeV}$. Recent strategies propose initial state radiation (ISR) and low $p_{T}$ leptons to probe this challenging region $[81,82]$, as adopted by the ATLAS $2 \ell$ ISR search [14]. Our strategy could gain sensitivity here if lepton trigger thresholds are lowered by using AFP and CT-PPS information, motivating future developments.

A striking feature of Fig. 3 is that our proposal decisively probes regions favored by DM and muon $(g-2)_{\mu}$ phenomenology. We evaluate these noncollider observables using MICROMEGAs v4.2.1 [80]. The gray dashed contour indicates where the $\tilde{\chi}_{1}^{0}$ relic abundance matches the Planck measurement $\Omega_{\tilde{\chi}_{1}^{0}} h^{2}=\Omega_{\mathrm{DM}}^{\text {Planck }} h^{2}=0.12 \quad$ [4]. Depletion of $\Omega_{\tilde{\chi}_{1}^{0}} h^{2}$ occurs via coannihilation processes such as $\tilde{\ell} \tilde{\chi}_{1}^{0} \rightarrow \ell \gamma$, whose rate grows exponentially $\sim e^{-\Delta m\left(\tilde{e}, \tilde{\chi}_{1}^{0}\right) / m(\tilde{\ell})}$ with smaller mass differences [5,6]. At low $m(\tilde{\ell})$, the self-annihilation via the $Z$ boson "funnel" becomes competitive, allowing larger mass splittings to satisfy $\Omega_{\mathrm{DM}}^{\text {Planck }} h^{2}$. Loop corrections from $\tilde{\ell}$ and $\tilde{\chi}_{1}^{0}$ states contribute to the muon anomalous magnetic moment $a_{\mu}=\frac{1}{2}(g-2)_{\mu}$. The green dashed line indicates modifications consistent with the measured discrepancy $\Delta a_{\mu}=$ $a_{\mu}^{\text {measured }}-a_{\mu}^{\text {predicted }} \simeq 2.5 \times 10^{-9}$ [8]. While we consider these features in a simplified model, the phenomenology is qualitatively consistent with those in global fits of more complete 11-parameter models [83].

If the fourfold mass degeneracy scheme is relaxed, the LHC blind corridor widens to $10 \lesssim \Delta m\left(\tilde{\mu}_{R}, \tilde{\chi}_{1}^{0}\right) \lesssim 90 \mathrm{GeV}$ [11-14], where our strategy will play an important role. In conventional electroweak production, the right-handed states $\tilde{\ell}_{R}$ have order 3 times smaller cross sections than the left-handed $\tilde{\ell}_{L}$ counterparts [84]. By contrast, the photon collider strategy has the advantage of equal QED cross sections for $\tilde{\ell}_{L}$ and $\tilde{\ell}_{R}$ states.

This proposal is widely extendable to other search channels and electrically charged targets. So-called $R$ parity violating scenarios where the $\tilde{\chi}_{1}^{0}$ decays to higher multiplicity final states can profit from clean events. Charged fermions (charginos) face similar difficulties discriminating against $W W$ backgrounds and may benefit in combination with a hadronic channel. Scalar quarks, charged Higgs bosons, spin 1 mediators, and disappearing track signatures are also motivating scenarios.

In summary, we proposed a search strategy using the LHC as a photon collider to open sensitivity beyond LEP in the challenging corridor $15 \lesssim \Delta m\left(\tilde{e}, \tilde{\chi}_{1}^{0}\right) \lesssim 60 \mathrm{GeV}$ favored by $\mathrm{DM}$ and $(g-2)_{\mu}$ phenomenology. Proton tagging enables the initial state and missing momentum four-vector $p_{\text {miss }}$ to be reconstructed, offering striking background discrimination inaccessible to current LHC searches. We encourage experimental collaborations to include this forward physics frontier in flagship hadron collider searches for DM and their charged mediators.
We thank the DM@LHC Workshop at Heidelberg University, where discussions for this work began, for the hospitality. We are grateful to Alan Barr for the helpful conversations and feedback on the manuscript. We also thank Jamie Boyd, Savannah Clawson, Till Eifert, Will Fawcett, Barak Gruberg, Lucian Harland-Lang, Valery Khoze, Tommaso Lari, Paul Newman, Christoph Paus, Andy Pilkington, Rafał Staszewski, and Marek Tasevsky for the useful discussions. L. B. is supported by St. John's College, Oxford. J. L. is supported by STFC.

*lydia.beresford@physics.ox.ac.uk jesse.liu@physics.ox.ac.uk

[1] H. Goldberg, Constraint on the Photino Mass from Cosmology, Phys. Rev. Lett. 50, 1419 (1983); Erratum, Phys. Rev. Lett. 103, 099905(E) (2009).

[2] J. R. Ellis, J. S. Hagelin, D. V. Nanopoulos, K. A. Olive, and M. Srednicki, Supersymmetric relics from the big bang, Nucl. Phys. B238, 453 (1984).

[3] G. Bertone, D. Hooper, and J. Silk, Particle dark matter: Evidence, candidates and constraints, Phys. Rep. 405, 279 (2005).

[4] Planck Collaboration, Planck 2018 results. VI. Cosmological parameters, arXiv:1807.06209.

[5] K. Griest and D. Seckel, Three exceptions in the calculation of relic abundances, Phys. Rev. D 43, 3191 (1991).

[6] J. Edsjo and P. Gondolo, Neutralino relic density including coannihilations, Phys. Rev. D 56, 1879 (1997).

[7] Muon $g-2$ Collaboration, Final report of the muon E821 anomalous magnetic moment measurement at BNL, Phys. Rev. D 73, 072003 (2006).

[8] T. Aoyama, M. Hayakawa, T. Kinoshita, and M. Nio, Complete Tenth-Order QED Contribution to the Muon $g-2$, Phys. Rev. Lett. 109, 111808 (2012).

[9] K. Hagiwara, R. Liao, A. D. Martin, D. Nomura, and T. Teubner, $(g-2)_{\mu}$ and $\alpha\left(M_{Z}^{2}\right)$ re-evaluated using new precise data, J. Phys. G 38, 085003 (2011).

[10] M. A. Ajaib, B. Dutta, T. Ghosh, I. Gogoladze, and Q. Shafi, Neutralinos and sleptons at the LHC in light of muon $(g-2)_{\mu}$, Phys. Rev. D 92, 075033 (2015).

[11] ATLAS Collaboration, Search for direct production of charginos, neutralinos and sleptons in final states with two leptons and missing transverse momentum in $p p$ collisions at $\sqrt{s}=8 \mathrm{TeV}$ with the ATLAS detector, J. High Energy Phys. 05 (2014) 071.

[12] ATLAS Collaboration, Search for electroweak production of supersymmetric particles in final states with two or three leptons at $\sqrt{s}=13 \mathrm{TeV}$ with the ATLAS detector, Eur. Phys. J. C 78, 995 (2018).

[13] CMS Collaboration, Search for supersymmetric partners of electrons and muons in proton-proton collisions at $\sqrt{s}=13$ TeV, Phys. Lett. B 790, 140 (2019).

[14] ATLAS Collaboration, Search for electroweak production of supersymmetric states in scenarios with compressed mass spectra at $\sqrt{s}=13 \mathrm{TeV}$ with the ATLAS detector, Phys. Rev. D 97, 052010 (2018). 
[15] ALEPH, DELPHI, L3, OPAL Experiments, Combined LEP Selectron/Smuon/Stau Results, 183-208 GeV, Technical Report No. LEPSUSYWG/04-01.1, 2004.

[16] ALEPH Collaboration, Search for scalar leptons in $e^{+} e^{-}$ collisions at center-of-mass energies up to $209 \mathrm{GeV}$, Phys. Lett. B 526, 206 (2002).

[17] L3 Collaboration, Search for scalar leptons and scalar quarks at LEP, Phys. Lett. B 580, 37 (2004).

[18] F. del Aguila and L. Ametller, On the detectability of sleptons at large hadron colliders, Phys. Lett. B 261, 326 (1991).

[19] H. Baer, C.-H. Chen, F. Paige, and X. Tata, Detecting sleptons at hadron colliders and supercolliders, Phys. Rev. D 49, 3283 (1994).

[20] H. Baer, C.-H. Chen, F. Paige, and X. Tata, Signals for minimal supergravity at the CERN Large Hadron Collider. II. Multilepton channels, Phys. Rev. D 53, 6241 (1996).

[21] J. Alwall, P. C. Schuster, and N. Toro, Simplified models for a first characterization of new physics at the LHC, Phys. Rev. D 79, 075020 (2009).

[22] J. Alwall, M. P. Le, M. Lisanti, and J. G. Wacker, Modelindependent jets plus missing energy searches, Phys. Rev. D 79, 015005 (2009).

[23] D. Alves et al. (LHC New Physics Working Group), Simplified models for LHC new physics searches, J. Phys. G 39, 105005 (2012).

[24] R. Essig, E. Izaguirre, J. Kaplan, and J. G. Wacker, Heavy flavor simplified models at the LHC, J. High Energy Phys. 01 (2012) 074.

[25] O. Buchmueller, M. J. Dolan, S. A. Malik, and C. McCabe, Characterising dark matter searches at colliders and direct detection experiments: Vector mediators, J. High Energy Phys. 01 (2015) 037.

[26] J. Abdallah et al., Simplified models for dark matter searches at the LHC, Phys. Dark Universe 9-10, 8 (2015).

[27] D. Abercrombie et al., Dark matter benchmark models for early LHC run-2 searches: Report of the ATLAS/CMS Dark Matter Forum, arXiv:1507.00966.

[28] ATLAS Collaboration, Search for dark matter and other new phenomena in events with an energetic jet and large missing transverse momentum using the ATLAS detector, J. High Energy Phys. 01 (2018) 126.

[29] ATLAS Collaboration, Search for new phenomena in dijet mass and angular distributions from $p p$ collisions at $\sqrt{s}=$ $13 \mathrm{TeV}$ with the ATLAS detector, Phys. Lett. B 754, 302 (2016).

[30] ATLAS Collaboration, Search for dark matter at $\sqrt{s}=$ $13 \mathrm{TeV}$ in final states containing an energetic photon and large missing transverse momentum with the ATLAS detector, Eur. Phys. J. C 77, 393 (2017).

[31] ATLAS Collaboration, Search for Dark Matter Produced in Association with a Higgs Boson Decaying to $b \bar{b}$ using $36 \mathrm{fb}^{-1}$ of $p p$ Collisions at $\sqrt{s}=13 \mathrm{TeV}$ with the ATLAS Detector, Phys. Rev. Lett. 119, 181804 (2017).

[32] B. Dutta, K. Fantahun, A. Fernando, T. Ghosh, J. Kumar, P. Sandick, P. Stengel, and J. W. Walker, Probing squeezed bino-slepton spectra with the Large Hadron Collider, Phys. Rev. D 96, 075037 (2017).

[33] K. Piotrzkowski, Tagging two photon production at the CERN LHC, Phys. Rev. D 63, 071502(R) (2001).
[34] ATLAS Collaboration, Technical design report for the ATLAS Forward Proton detector, Technical Reports No. CERN-LHCC-2015-009 and No. ATLAS-TDR-024, 2015.

[35] M. Albrow et al. (CMS and TOTEM diffractive and Forward Physics Working Group), Prospects for diffractive and forward physics at the LHC, Technical Report No. CERN-LHCC-2006-039-G-124.

[36] CMS and TOTEM Collaborations, CMS-TOTEM precision proton spectrometer technical design report, Technical Report No. CERN-LHCC-2014-021, TOTEMTDR-003, CMS-TDR-13, 2014.

[37] CMS and TOTEM Collaborations, Observation of protontagged, central (semi)exclusive production of high-mass lepton pairs in $p p$ collisions at $13 \mathrm{TeV}$ with the CMSTOTEM precision proton spectrometer, J. High Energy Phys. 07 (2018) 153.

[38] D. d'Enterria and G. G. da Silveira, Observing Light-ByLight Scattering at the Large Hadron Collider, Phys. Rev. Lett. 111, 080405 (2013); Erratum, Phys. Rev. Lett. 116, 129901(E) (2016).

[39] ATLAS Collaboration, Evidence for light-by-light scattering in heavy-ion collisions with the ATLAS detector at the LHC, Nat. Phys. 13, 852 (2017).

[40] E. Chapon, C. Royon, and O. Kepka, Anomalous quartic $W W \gamma \gamma, Z Z \gamma \gamma$, and trilinear $W W \gamma$ couplings in two-photon processes at high luminosity at the LHC, Phys. Rev. D 81, 074003 (2010).

[41] S. Fichet, G. von Gersdorff, O. Kepka, B. Lenzi, C. Royon, and M. Saimpert, Probing new physics in diphoton production with proton tagging at the Large Hadron Collider, Phys. Rev. D 89, 114004 (2014).

[42] S. Knapen, T. Lin, H. K. Lou, and T. Melia, Searching for Axionlike Particles with Ultraperipheral Heavy-Ion Collisions, Phys. Rev. Lett. 118, 171801 (2017).

[43] C. Baldenegro, S. Fichet, G. von Gersdorff, and C. Royon, Searching for axion-like particles with proton tagging at the LHC, J. High Energy Phys. 06 (2018) 131.

[44] Sylvain Fichet, Shining light on polarizable dark particles, J. High Energy Phys. 04 (2017) 088.

[45] V. A. Khoze, A. D. Martin, and M. G. Ryskin, Can invisible objects be seen via forward proton detectors at the LHC?, J. Phys. G 44, 055002 (2017).

[46] J. Ohnemus, T. F. Walsh, and P. M. Zerwas, $\gamma \gamma$ production of non-strongly interacting SUSY particles at hadron colliders, Phys. Lett. B 328, 369 (1994).

[47] N. Schul and K. Piotrzkowski, Detection of two-photon exclusive production of supersymmetric pairs at the LHC, Nucl. Phys. B, Proc. Suppl. 179-180, 289 (2008).

[48] FP420 R \& D Collaboration, The FP420 R \& D Project: Higgs and new physics with forward protons at the LHC, J. Instrum. 4, T10001 (2009).

[49] J. de Favereau de Jeneret, V. Lemaitre, Y. Liu, S. Ovyn, T. Pierzchala, K. Piotrzkowski, X. Rouby, N. Schul, and M. Vander Donckt, High energy photon interactions at the LHC, arXiv:0908.2020.

[50] L. A. Harland-Lang, C. H. Kom, K. Sakurai, and W. J. Stirling, Measuring the masses of a pair of semi-invisibly decaying particles in central exclusive production with forward proton tagging, Eur. Phys. J. C 72, 1969 (2012). 
[51] V. M. Budnev, I. F. Ginzburg, G. V. Meledin, and V. G. Serbo, The two photon particle production mechanism. Physical problems. Applications. Equivalent photon approximation, Phys. Rep. 15, 181 (1975).

[52] S. J. Brodsky, T. Kinoshita, and H. Terazawa, Two photon mechanism of particle production by high-energy colliding beams, Phys. Rev. D 4, 1532 (1971).

[53] G. Tupper and M. A. Samuel, $W^{+} W^{-}$pair production in two-photon collisions and the magnetic moment of the $W^{ \pm}$ bosons, Phys. Rev. D 23, 1933 (1981).

[54] J. Alwall, M. Herquet, F. Maltoni, O. Mattelaer, and T. Stelzer, MadGraph 5: Going beyond, J. High Energy Phys. 06 (2011) 128.

[55] J. Alwall, R. Frederix, S. Frixione, V. Hirschi, F. Maltoni, O. Mattelaer, H.-S. Shao, T. Stelzer, P. Torrielli, and M. Zaro, The automated computation of tree-level and next-toleading order differential cross sections, and their matching to parton shower simulations, J. High Energy Phys. 07 (2014) 079.

[56] L. Heinrich, LUKASHEINRICH/PYLHE v0.0.4, https://doi.org// 10.5281/zenodo.1217032, 2018.

[57] See Supplemental Material at http://link.aps.org/ supplemental/10.1103/PhysRevLett.123.141801 for details of selection efficiency, soft survival probabilities, forward detector acceptance, and pileup mitigation strategies.

[58] V. A. Khoze, A. D. Martin, and M. G. Ryskin, Prospects for new physics observations in diffractive processes at the LHC and tevatron, Eur. Phys. J. C 23, 311 (2002).

[59] V. A. Khoze, A. D. Martin, and M. G. Ryskin, Diffraction at the LHC, Eur. Phys. J. C 73, 2503 (2013).

[60] L. A. Harland-Lang, V. A. Khoze, and M. G. Ryskin, Exclusive LHC physics with heavy ions: SuperChic 3, Eur. Phys. J. C 79, 39 (2019).

[61] ATLAS Collaboration, Studies of the performance of the ATLAS detector using cosmic-ray muons, Eur. Phys. J. C 71, 1593 (2011).

[62] J. de Favereau, C. Delaere, P. Demin, A. Giammanco, V. Lematre, A. Mertens, and M. Selvaggi (DELPHES 3 Collaboration), DELPHES 3, A modular framework for fast simulation of a generic collider experiment, J. High Energy Phys. 02 (2014) 057.

[63] A. J. Barr, Measuring slepton spin at the LHC, J. High Energy Phys. 02 (2006) 042.

[64] T. Melia, Spin before mass at the LHC, J. High Energy Phys. 01 (2012) 143.

[65] C. G. Lester and D. J. Summers, Measuring masses of semiinvisibly decaying particles pair produced at hadron colliders, Phys. Lett. B 463, 99 (1999).

[66] A. Barr, C. Lester, and P. Stephens, A variable for measuring masses at hadron colliders when missing energy is expected; $m_{\mathrm{T} 2}$ : The truth behind the glamour, J. Phys. G 29, 2343 (2003).

[67] C. G. Lester and B. Nachman, Bisection-based asymmetric $\mathrm{M}_{T 2}$ computation: A higher precision calculator than existing symmetric methods, J. High Energy Phys. 03 (2015) 100.
[68] ATLAS Collaboration, Measurement of the exclusive $\gamma \gamma \rightarrow \mu^{+} \mu^{-}$process in proton-proton collisions at $\sqrt{s}=$ $13 \mathrm{TeV}$ with the ATLAS detector, Phys. Lett. B 777, 303 (2018).

[69] ATLAS Collaboration, Measurement of exclusive $\gamma \gamma \rightarrow$ $W^{+} W^{-}$production and search for exclusive Higgs boson production in $p p$ collisions at $\sqrt{s}=8 \mathrm{TeV}$ using the ATLAS detector, Phys. Rev. D 94, 032011 (2016).

[70] CMS Collaboration, Evidence for exclusive $\gamma \gamma \rightarrow W^{+} W^{-}$ production and constraints on anomalous quartic gauge couplings in $p p$ collisions at $\sqrt{s}=7$ and $8 \mathrm{TeV}$, J. High Energy Phys. 08 (2016) 119.

[71] ATLAS Collaboration, Zero degree calorimeters for ATLAS, Technical Report No. CERN-LHCC-2007-01, 2007.

[72] M. W. Phipps, A new ATLAS ZDC for the high radiation environment at the LHC, Technical Report No. ATL-FWDSLIDE-2018-288, 2018.

[73] C. Royon, M. Saimpert, O. Kepka, and R. Zlebcik, Timing detectors for proton tagging at the LHC, Acta Phys. Pol. B 7, 735 (2014).

[74] L. Bonnet, J. Liao, and K. Piotrzkowski, Study on GASTOFA 10 ps resolution timing detector, Nucl. Instrum. Methods Phys. Res., Sect. A 762, 77 (2014).

[75] L. A. Harland-Lang, V. A. Khoze, M. G. Ryskin, and M. Tasevsky, LHC searches for dark matter in compressed mass scenarios: Challenges in the forward proton mode, J. High Energy Phys. 04 (2019) 010.

[76] R. Staszewski and J. Chwastowski, Timing detectors for forward physics, Nucl. Instrum. Methods Phys. Res., Sect. A 940, 45 (2019).

[77] ATLAS Collaboration, Prospects and results from the AFP detector in ATLAS, Report No. ATL-FWD-SLIDE-2019165, 2019.

[78] G. Cowan, Discovery sensitivity for a counting experiment with background uncertainty, www.pp.rhul.ac.uk/ cowan/ stat/medsig/medsigNote.pdf.

[79] G. Cowan, K. Cranmer, E. Gross, and O. Vitells, Asymptotic formulae for likelihood-based tests of new physics, Eur. Phys. J. C 71, 1554 (2011); Erratum, Eur. Phys. J. C 73, 2501(E) (2013).

[80] G. Blanger, F. Boudjema, A. Pukhov, and A. Semenov, MICROMEGAs 4.1: Two dark matter candidates, Comput. Phys. Commun. 192, 322 (2015).

[81] Z. Han and Y. Liu, $M_{\mathrm{T} 2}$ to the rescue: Searching for sleptons in compressed spectra at the LHC, Phys. Rev. D 92, 015010 (2015).

[82] A. Barr and J. Scoville, A boost for the EW SUSY hunt: Monojet-like search for compressed sleptons at LHC14 with $100 \mathrm{fb}^{1}$, J. High Energy Phys. 04 (2015) 147.

[83] E. Bagnaschi et al., Likelihood analysis of the pMSSM11 in light of LHC 13-TeV data, Eur. Phys. J. C 78, 256 (2018).

[84] B. Fuks, M. Klasen, D. R. Lamprea, and M. Rothering, Revisiting slepton pair production at the Large Hadron Collider, J. High Energy Phys. 01 (2014) 168. 